\title{
Design and Analysis of a Photonic Crystal Based Biosensor Working at THz Frequency Region
}

\author{
Bratati Ghosh, Shukufe Rahman, Ahsan Habib and Subrata Das* \\ Department of Applied Physics, Electronics and Communication Engineering, Dhaka University, Dhaka-1000, Bangladesh
}

(Received: 28 August 2012; Accepted: 4 June 2013)

\begin{abstract}
2D Photonic Crystal is investigated to find photonic band gap for the purpose of biosensing at $\mathrm{THz}$ frequency region. Several structures, one consisting of air holes in Si background and the others consisting of air holes filled with different analytes of having different Refractive Index (RI) in same Si background are considered. For each structure the change in photonic band gap due to the change in RI is observed and shown graphically. By comparing this plot with a standard chart of RI of different materials, any unknown biological analyte can be identified. Thus a biosensing method is developed by using the relationship between RI and band gap of photonic crystals. By comparing and calculating from the relationship; the maximum sensitivity of the developed biosensor is found to be $66.6 \%$.
\end{abstract}

Keywords: Biosensor, Photonic Crystal, THz frequency range

\section{Introduction}

A biosensor is an analytical device for the detection of an analyte that combines a biological component with a physiochemical detector component. The term 'biosensor' is often used to cover sensor devices used in order to determine the concentration of substances and other parameters of biological interest even where they do not utilize a biological system directly ${ }^{1}$. Photonic crystal (PC) biosensors are a novel type of label-free optical biosensing platform. Photonic crystals are composed of periodic dielectric or metallo-dielectric nanostructures that affect the propagation of electromagnetic waves (EM waves) in the same way as the periodic potential in a semiconductor crystal affects the electron motion by defining allowed and forbidden electronic energy bands ${ }^{2}$. Essentially, photonic crystals contain regularly repeating internal regions of high and low dielectric constant. Photons (behaving as waves) propagate through this structure - or not - depend on their wavelength. Through the proper application of PC design and fabrication, electromagnetic fields may be confined and concentrated to enhance the interaction between light and biological material in contact with the $\mathrm{PC}$ which is efficient for optical biosensor.

Biosensors at $\mathrm{THz}$ region are nowadays technologically hot topics due to the possible applications in such as medical diagnostic and health care. Within this region of spectrum, most of the biomolecules show characteristic fingerprints. In literature $^{3} \mathrm{THz}$ region is well acceptably defined as the regime lies between microwave and optical frequencies, (0.1-15 THz), commonly known as $\mathrm{THz}$ gap. The $\mathrm{THz}$ radiation has the ability to penetrate deep into many organic materials without the damage as opposed to ionizing radiation such as X-rays. Also, because $\mathrm{THz}$ is readily absorbed by water, it can be used to distinguish between materials with varying water content ${ }^{4}$.

At present several researches are working on biosensors in the $\mathrm{THz}$ frequency range. A new class of disposable, microplate-based optical biosensors capable of detecting protein-DNA interactions has been developed by a group of scientist of University of Illinois at Urbana ${ }^{5}$. Utilizing

*Author for Correspondence. e-mail: uromman@yahoo.com photonic crystal for enhanced biosensing ${ }^{6}$, where computer models are used to predict the reflectance spectra and sensitivity performance of a one-dimensional photonic crystal biosensor. The propagation of terahertz waves in two-dimensional photonic-crystal waveguides to investigate the effects of introducing small quantities of molecules into selected air holes for sensor applications is carried out by Hamza Kurt and D. S. Citrin ${ }^{7}$. In this present work, change in the bandgap of 2D hexagonal PC with circular air hole in Si substrate filled with different analytes is investigated to find the refractive index of the analytes. For bandgap calculation 'PWE band solver' of the software package OPTIFDTD $^{\mathrm{TM}}$ is used ${ }^{8}$. The PC structure is constructed by creating air holes onto a dielectric substrate. The air holes will act as a container for the analytes. After filling the air holes by analyte of different RI, the band gap structure is observed.

\section{Modeling}

\section{(a) Lattice Formation}

Silicon (Si) with a refractive index of 3.24 has been considered as the dielectric material. Circular air holes with radius, $\mathrm{r}=0.48 \mathrm{a}(\mathrm{a}=$ lattice constant in $\mu \mathrm{m})$ are perforated on the $\mathrm{Si}$ substrate in a hexagonal periodicity. The wafer dimension is taken to be $8 \times 8 \mu \mathrm{m}$. Figure 1 shows the considered structure with the profile of refractive index variation.

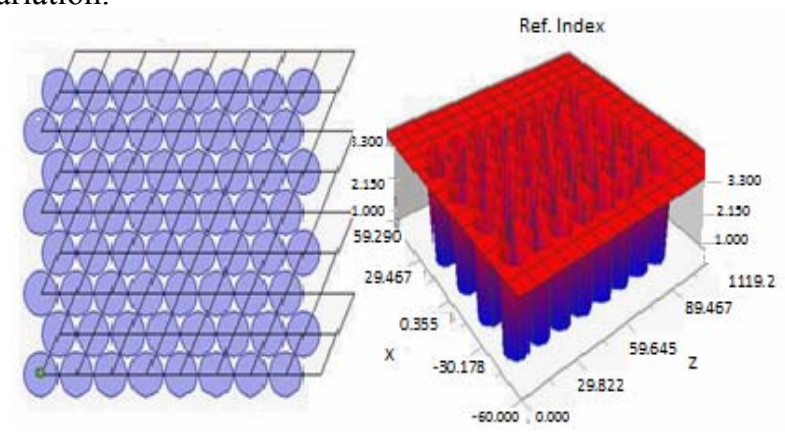

(a)

(b)

Fig. 1. (a) hexagonal lattice of circular air holes in dielectric background (b) refractive index profile of the crystal structure. 


\section{(b)Determination of Photonic Bandgap}

The photonic band gap structure is found by PWE simulator of OPTIFDTD ${ }^{\mathrm{TM}}$. The polarization of the source signal is defined as TE polarization. The lattice constant, ' $\mathrm{a}$ ' is arbitrarily chosen to be $1 \mu \mathrm{m}$. After the simulation the band structure is found as shown in Fig 2. The achieved bandgaps in the PC structure are summarizes in Table 1.

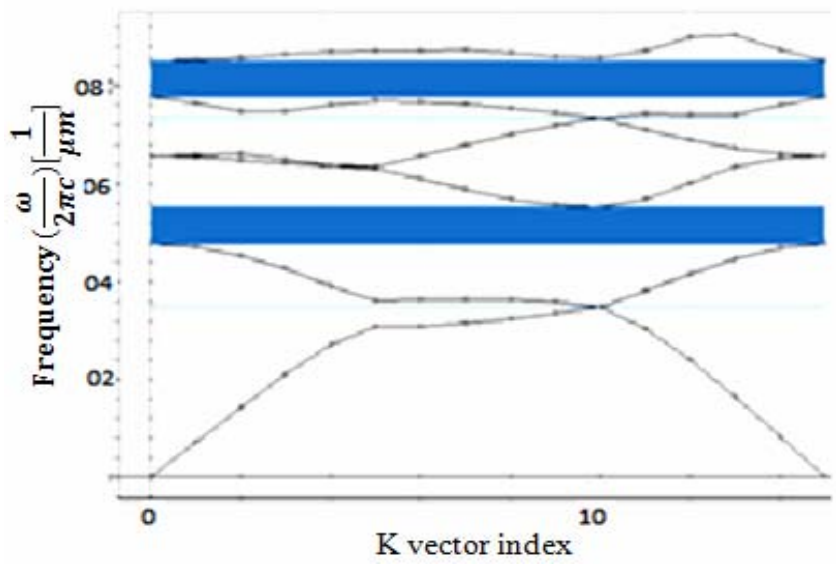

Fig. 2. Bandgap for circular air hole in Si Background

Table 1. Band gap for circular air hole in Si Background

\begin{tabular}{|l|l|l|l|}
\hline $\begin{array}{c}\text { No of } \\
\text { bandgap }\end{array}$ & $\begin{array}{c}\text { Lower } \\
\text { limit(a/ } \Lambda) \\
\left(\frac{1}{\mu m}\right)\end{array}$ & $\begin{array}{c}\text { Upper } \\
\text { limit(a/ } \Lambda) \\
\left(\frac{1}{\mu m}\right)\end{array}$ & $\begin{array}{c}\text { Bandgap }) \\
(\mathrm{a} / \Lambda)\left(\frac{1}{\mu m}\right)\end{array}$ \\
\hline 1 & 0.348838 & 0.348839 & $1.2 \times 10^{-7}$ \\
\hline 2 & 0.047611 & 0.551704 & $7.55955 \times 10^{-2}$ \\
\hline 3 & 0.73355 & 0.733721 & $1.7103 \times 10^{-4}$ \\
\hline 4 & 0.779566 & 0.851592 & $7.20256 \times 10^{-2}$ \\
\hline
\end{tabular}

As the frequency of interest is in $\mathrm{THz}$ range we need to obtain the band gap at $\mathrm{THz}$ region. For this purpose the scaling property of Maxwell's equation is utilized ${ }^{9}$. As a result the lattice constant ' $a$ ' of the considered periodic structure has been changed. The calculation is shown in Equation 1 for the lower limit frequency $0.779566(\mathrm{a} / \lambda)$ of 4th band gap

$$
a=\frac{e}{f} \times 0.779566
$$

Where, $\mathrm{a}=$ lattice periodicity, $\mathrm{c}=$ speed of light, $\mathrm{f}=$ frequency.

If $f=19 \mathrm{THz}$ is considered as the center frequency, then from Equation (1) the value of ' $a$ ' is obtained as 12 . Next, the air holes are filled with different analytes of different Refractive Index. As the Refractive Index varies the band gap also varies. The same PC structure is examined for five more cases with five different analytes. Table 2 summarizes the band gap for analytes with different refractive index.
Table 2. Resultant band gap for different analytes

\begin{tabular}{|l|l|}
\hline RI of analytes & Band gap (THz) \\
\hline 1 & $11.8 \times 10^{-2}$ \\
\hline 1.15 & $7.86 \times 10^{-2}$ \\
\hline 1.2 & $5.34 \times 10^{-2}$ \\
\hline 1.25 & $3.21 \times 10^{-2}$ \\
\hline 1.3 & $1.382 \times 10^{-2}$ \\
\hline 1.33 & $4.2 \times 10^{-3}$ \\
\hline
\end{tabular}

\section{Result and Discussion}

Using the values from Table 2 a graph of RI Vs bandgap for different analytes is shown in Figure 3, where the values of Refractive Index (RI) is plotted along $X$ axis and the corresponding band gap value along $\mathrm{Y}$ axis. From the graph in Figure 3, it is obvious that the increasing value of RI of the analyte is accounted for the sharp decrease in band gap.

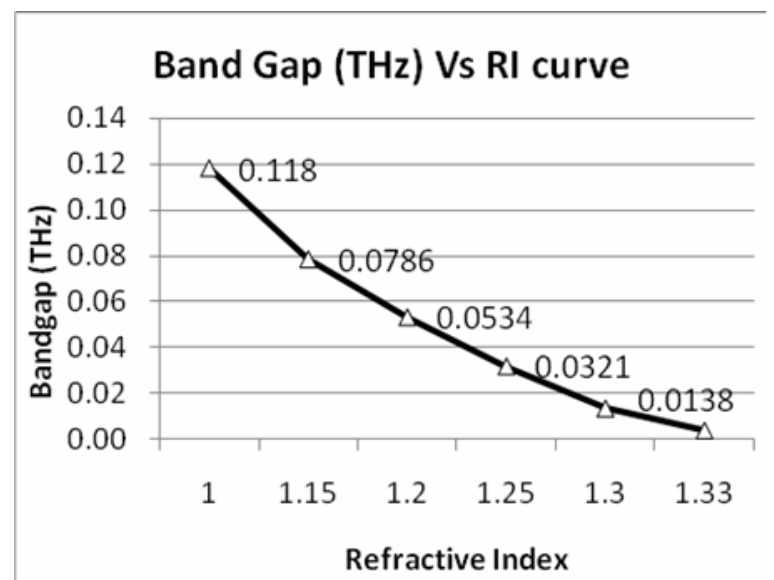

Fig.3. Refractive index Vs band gap variation for different analytes

Now one can identify any unknown analyte by measuring the change in RI for that analyte. If we fill the air holes by unknown analyte a change in band gap will be found. If the change in band gap is within $0-0.15 \mathrm{THz}$ of frequency range, then from the graph the respective $\mathrm{RI}$ of that analyte can be found. After finding the RI of the analyte, from a standard chart where the RI of different material is given, that analyte can be identified.

\section{Sensitivity Analysis}

Now we can analyze the sensitivity of the proposed biosensor. The change in band gap due to the variation in refractive index (RI) has been counted. From the response curve of Figure 3, it is visible that the variation is not linear. So the total curve is divided into three separate regions and the sensitivity is calculated for each region.

1) First we consider the region where the band gap changes from 0.12 to 0.1 and the RI changes from 1 to 1.03 , then Sensitivity $=|(0.1-0.12) /(1.03-1)| \times 100 \%=66.6 \%$. So at this region, a sharp sensitivity is observed. 
2) The second region is taken for the RI changes from 1.2 to 1.25 and the band gap changes from 0.0534 to 0.0321 . In this region the sensitivity can be calculated as, Sensitivity $=$ $|(0.0321-0.0524) /(1.25-1.2)| \times 100 \%=40.6 \%$. Here, the sensitivity decreases from that of the first region.

3) For the third region, RI changes from 1.3 to 1.33 and the band gap changes from 0.01382 to 0.0042 . So for this region sensitivity can be calculated as, | (0.0042-0.01382)/ (1.33$1.3) \mid \times 100 \%=32.07 \%$. In this region the sensitivity is much lower, almost half of that of the first region. That means as the RI of the analyte increases, the sensitivity decreases gradually.

\section{Conclusion}

An investigation for the bandgap structures of $2 \mathrm{D}$ photonic crystals with hexagonal lattice of air holes is performed. Then after filling the air holes with different analytes of different RI the shift in band gap is observed. It is found that as the RI of the analytes increases, there is a sharp change in bandgap. So if we want to identify any biological analyte, we can find the RI of that analyte by following the change in band gap at $\mathrm{THz}$ region. Thus photonic crystal can be used as biosensor at $\mathrm{THz}$ region.

\section{Reference}

1. Mohanty S. P. and E. Kougianos, 2006. Biosensors: A Tutorial Review. IEEE Potentials, 25(2), 35-40.
2. Joannopoulas J. D., P. R. Villeneuve and S. Fan, 1997. Photonic Crystals: putting a new twist on light, Nature.386, $143-149$.

3. Mueller E. R., 2001. Terahertz Radiation: Applications and Sources. American Institute of Physics, INPHFA, 9(4), 27

4. Chamberlain J. M. and R.E. Miles, 1997. New Direction in Terahertz Technology. in NATO ASI Series, Kluwer Academic Publishers, ISBN 0-7923-4537-1.

5. Chan L. L. et al, 2008. A General Method for Discovering Inhibitors of Protein DNA Interactions Using Photonic Crystal Biosensors. American Chemical Society, Chemical Biology, 3(7), 437-448.

6. Block I. D. et al, 2006. Photonic crystal optical biosensor incorporating structured low-index porous dielectric. Sensors and ActuatorsB:Chemical,120,187-193.

7. Kurt H. and D.S. Citrin, 2005. Photonic crystals for biochemical sensing in the terahertz region. Applied Physics Letters, 87(4).

8. OptiFDTD Technical background and tutorials (version 8.1), 2009.

9. Joannopoulas J.D. et al, 2008. Photonic Crystals Molding of Flow of Light. $2^{\text {nd }}$ Edition, Princeton University Press. 
Bratati Ghosh, Shukufe Rahman, Ahsan Habib, and Subrata Das 
